\title{
Clinical Disorders in a Post War British Cohort Reaching Retirement: Evidence from the First National Birth Cohort Study
}

\author{
Mary B. Pierce ${ }^{1 *}$, Richard J. Silverwood ${ }^{2}$, Dorothea Nitsch ${ }^{2}$, Judith E. Adams ${ }^{3}$, Alison M. Stephen ${ }^{4}$, \\ Wing Nip ${ }^{4}$, Peter Macfarlane ${ }^{5}$, Andrew Wong ${ }^{1}$, Marcus Richards ${ }^{1}$, Rebecca Hardy ${ }^{1}$, Diana Kuh ${ }^{1}$, on behalf \\ of the NSHD Scientific and Data Collection Teams
}

1 MRC Unit for Lifelong Health \& Ageing, London, England, 2 Department of Non-Communicable Disease Epidemiology, London School of Hygiene and Tropical Medicine, London, England, 3 Manchester Academic Health Science Centre, University of Manchester, Manchester, England, 4 MRC Human Nutrition Research, Elsie Widdowson Laboratory, Cambridge, England, 5 Electrocardiology Section, Royal Infirmary, University of Glasgow, Glasgow, Scotland

\begin{abstract}
Background: The medical needs of older people are growing because the proportion of the older population is increasing and disease boundaries are widening. This study describes the distribution and clustering of 15 common clinical disorders requiring medical treatment or supervision in a representative British cohort approaching retirement, and how health tracked across adulthood.

Methods and Findings: The data come from a cohort of 2661 men and women, 84\% of the target sample, followed since birth in England, Scotland and Wales in 1946, and assessed at 60-64 years for: cardio and cerebro-vascular disease, hypertension, raised cholesterol, renal impairment, diabetes, obesity, hypothyroidism, hyperthyroidism, anaemia, respiratory disease, liver disease, psychiatric problems, cancers, atrial fibrillation on ECG and osteoporosis. We calculated the proportions disorder-free, with one or more disorders, and the level of undiagnosed disorders; and how these disorders cluster into latent classes and relate to health assessed at 36 years. Participants had, on average, two disorders (range 0-9); only $15 \%$ were disorder-free. The commonest disorders were hypertension $(54.3 \%, 95 \% \mathrm{Cl} 51.8 \%-56.7 \%)$, obesity $(31.1 \%$, $28.8 \%-33.5 \%)$, raised cholesterol $(25.6 \%, 23.1-28.26 \%)$, and diabetes or impaired fasting glucose $(25.0 \%, 22.6-27.5 \%)$. A cluster of one in five individuals had a high probability of cardio-metabolic disorders and were twice as likely than others to have been in the poorest health at 36 years. The main limitations are that the native born sample is entirely white, and a combination of clinical assessments and self reports were used.

Conclusions: Most British people reaching retirement already have clinical disorders requiring medical supervision. Widening disease definitions and the move from a disease-based to a risk-based medical model will increase pressure on health services. The promotion of healthy ageing should start earlier in life and consider the individual's ability to adapt to and self manage changes in health.
\end{abstract}

Citation: Pierce MB, Silverwood RJ, Nitsch D, Adams JE, Stephen AM, et al. (2012) Clinical Disorders in a Post War British Cohort Reaching Retirement: Evidence from the First National Birth Cohort Study. PLoS ONE 7(9): e44857. doi:10.1371/journal.pone.0044857

Editor: Antony Bayer, Cardiff University, United Kingdom

Received May 31, 2012; Accepted August 7, 2012; Published September 19, 2012

Copyright: (C) 2012 Pierce et al. This is an open-access article distributed under the terms of the Creative Commons Attribution License, which permits unrestricted use, distribution, and reproduction in any medium, provided the original author and source are credited.

Funding: This work was supported by the Medical Research Council grant numbers U1200632239 and U123092720. The funders had no role in study design, data collection and analysis, decision to publish, or preparation of the manuscript.

Competing Interests: The authors have declared that no competing interests exist.

*E-mail: m.pierce@nshd.mrc.ac.uk

\section{Introduction}

There is a longstanding and ongoing debate about the widening boundaries of disease and the costs and benefits of the medicalisation of health [1-5]. This debate is particularly relevant for the very old where multiple morbidity and polypharmacy are common and complicate treatment planning [6], and where medical problems may be overlooked or ignored [2]. It is also relevant for the early detection of disease at younger ages; for example, recent recommendations to screen everyone over 45 years for risk factors for cardiovascular disease [7] or prescribe statins or some form of 'polypill' for all those over 55 years old [8] would increase the proportion of the population with a disorder label and requiring regular medical supervision.

This debate needs to be informed about the current health status and treatment of older people. For the oldest old, in England, the Newcastle 85+ cohort [9] provides a description of their health status and treatment, and shows that nobody in this sample was disease free. National health surveys [10-12] also provide relevant information about the older population but they typically focus on different aspects of health in different years; and because they consist of a different population sample each year, it is impossible to estimate the extent of multiple morbidity. Moreover there is no way of defining in these surveys who is 'well' in the sense of being disease free. 
In a national sample of the British population reaching retirement age, we investigate the extent of common clinical disorders, defined as an impairment of body system or structure for which there is evidence or a consensus for medical intervention, in terms of active monitoring or treatment. The data come from the Medical Research Council (MRG) National Survey of Health and Development (NSHD), a birth cohort study of men and women born in 1946 and followed ever since [13,14]. The generation that this cohort represents is important to study, being the first of the post World War II baby boom, and benefitting from increased longevity compared with earlier cohorts. This generation will contribute to the growing proportion of the population aged 65 years and over, which is expected to rise in the UK by $32 \%$ from 11.8 million in 2008 to reach 15.6 million by 2033 [15]. The health of the baby boomers as they age will dominate the work of the health and social care systems for the next three decades, not only in the UK but in all Western developed nations.

In this cohort aged 60-64 years, we describe: the proportions with, and sex differences in, fifteen common clinical disorders of later life; the extent to which these clinical disorders are already diagnosed and/or treated; how these disorders are distributed and relate to current self reported health; and how these disorders cluster into latent classes and relate to health status previously assessed at age 36 years.

\section{Methods}

\section{Ethics statement}

The study protocol received ethical approval from the Central Manchester Research Ethics Committee for data collection taking place in Manchester, Birmingham, Cardiff and London. Ethical permission was given by the Scotland A Research Ethics Committee for the data collection taking place in Edinburgh. Written informed consent was obtained from the study member at each stage of data collection.

\section{Participants}

The MRG NSHD is a socially stratified sample of 5362 singleton children born in one week in March 1946 in England, Scotland and Wales [14]. From an initial maternity survey in 1946 [16], the sample consisted of all single births to married women with husbands in non-manual and agricultural employment and one in four of all comparable births to women with husbands in manual employment. Of the 5362 original study members, the study team was still in contact with $3163(59 \%)$ at age 60-64 years; $718(13.4 \%)$ had died, $594(11.1 \%)$ had previously withdrawn from the study, $567(10.6 \%)$ lived abroad and $320(5.9 \%)$ had been untraceable for more than ten years.

Study members received postal questionnaires between 2007 and 2008 and were invited for clinic visits between October 2007 and February 2011. If study members were unable or unwilling to come to one of the six Clinical Research Facilities (Edinburgh, Manchester, Cardiff, Birmingham, UCLH London or St Thomas' London) they were offered a slightly less comprehensive examination carried out in their own home by a trained nurse.

Of the 3163 people in the target sample, information was obtained from the postal questionnaire and/or visits from 2661 $(84 \%)$. Of these, 2462 people completed the postal questionnaire and 2229 had a visit, of whom 1690 attended a clinic and 539 had a home visit.

\section{Self-reported disorders}

For stroke, diabetes, cancer, angina, myocardial infarction (MI) and thyroid disease we went back to the first occasion on which participants were asked if they had ever had a doctor diagnosis of these conditions. These reports were updated at subsequent data collections. In 1989, participants were first asked if they had ever been told by a doctor that they had had a stroke, diabetes or cancer; in 1999 a similar question referred to angina, MI and thyroid disease; and in the most recent data collection questions on coronary artery bypass graft, angioplasty or stent were included. Cancers were also picked up from registrations on the NHS Information Centre Registry.

\section{Symptom scales}

At age 60-64 years participants completed postal versions of the MRC Chronic Bronchitis [17] and the Rose Angina and Intermittent Claudication [18] questionnaires. The General Health Questionnaire (GHQ-28) [19] which asks about affective symptoms was self completed during the clinic or home visit.

\section{Prescribed medications}

As part of the postal questionnaire, participants were asked to list all their prescribed medications, and the reasons why these were prescribed. These were checked by the nurses and used to help define some disorders (see below).

\section{Clinical assessments}

Full details of the research protocol can be found elsewhere [14] but brief descriptions are given below. Those visited by a nurse at home received the same assessment except for the scans.

Cardiovascular assessment. Two measures of systolic and diastolic blood pressure were taken with an OMROM 705 with the participant seated, with the second reading used in this analysis. Participants visiting the clinic also had a 12 lead electrocardiogram recorded (Burdick Eclipse 850i). The output was sent by modem to the ECG Core Laboratory at the University of Glasgow for reporting.

DXA bone scans. Bone density scans were performed in the lumbar spine (L1-4) and proximal femur (femoral neck, total hip) using Hologic QDR 4500 Discovery scanners (Hologic Inc., Bedford MA, USA) according to standard protocols developed by Prof. J. Adams. Scans were sent to the central core laboratory in the University of Manchester for analysis.

Blood sample. Participants were asked to fast from 22.00 hours on the night before the visit, and the majority of blood samples were collected between 08.00 and 09.00 hours the following day. Samples were processed on site according to standardised protocols. Full blood counts were undertaken at the local hospital on the day of the visit. Aliquots were dispatched to Addenbrooke's Hospital Cambridge for HbAlc to be measured.

The remaining aliquots were stored at $-80^{\circ} \mathrm{C}$ and couriered monthly, on dry ice, to the MRG Human Nutrition Research (HNR) laboratory in Cambridge. Serum lipids, fasting blood glucose, liver function tests, and serum creatinine were measured at HNR, and thyroid function tests at Addenbrooke's Hospital.

Serum total cholesterol, HDL cholesterol, triglycerides, glucose, and all liver function test parameters were measured colorimetrically on a Siemens Dimension Xpand analyser. LDL cholesterol was calculated from total cholesterol. HbAlc was analysed using the TOSOH G7 HPLC system. TSH and Free T4 were analysed using the Siemens Centaur Automated Immunoassay System. Free T3 was analysed using the Perkin Elmer AutoDELFIA Automated Immunoassay System until June 2009 and thereafter using the 
Siemens Centaur. Serum creatinine measurements were carried out using the Jaffe method.

\section{Defining the clinical disorders}

We assessed 15 clinical disorders expected in people of this age group: cardio/cerebro-vascular disease, hypertension, raised cholesterol, renal impairment, diabetes, obesity, hypothyroidism, hyperthyroidism, anaemia, respiratory disease, liver disease, psychiatric problems, cancers, ECG abnormalities, and osteoporosis.

Clinical disorder status was derived from one or more of: a selfreport of a doctor diagnosis of disease in response to a specific question; being positive on a validated scale for the disorder; a clinical assessment; currently receiving prescribed treatment for the disorder; or cancer registrations. The data sources and definitions for each clinical disorder are given in Table 1 [1727]. For some conditions (hypertension, raised cholesterol, renal impairment, diabetes, obesity, hypothyroidism, and hyperthyroidism) two levels of disorder were defined, level 1 where intervention is required irrespective of other information and level 2 where the type of intervention may depend on other information. For example, whether or not to treat a person with a raised cholesterol level depends on the overall 10 year risk of a significant event.

\section{Covariates}

Health status at 36 years. Cohort members had previously been identified as being in the best of health $(10 \%)$, intermediate $(65 \%)$ or worst of health $(25 \%)$ at age 36 years on the basis of measured blood pressure, lung function and body weight, self reported health problems and disability, and recent hospital admission [28].

Self-reported health status at 60-64 years. At age 60-64 years participants were asked to rate their current health on a 5 point scale from excellent to poor.

\section{Statistical methods}

The proportion with each clinical disorder was calculated as the number of participants meeting the relevant definition (see Table 1) divided by the number of participants with valid data for that measure. Where more than one measure was used in the definition of a disorder (for example total:HDL cholesterol ratio and reported statin/fibrate use for level 1 raised cholesterol), the sample was restricted to those with valid data for all relevant measures. The proportions were weighted to account for the social stratification in the original sample. Unweighted proportions were also calculated for comparison.

Undiagnosed disease. The number of participants who had diabetes, osteoporosis, hypertension, and thyroid disorders on examination, but who had not reported a doctor diagnosis of that condition or who were not on treatment for that specific disorder was divided by the total number of participants, to give the proportion of the total sample with undiagnosed disease. The proportion of those undiagnosed within the sample who had each of these disorders was also calculated. Social class-weighted results are presented, but unweighted results were produced for comparison.

Distribution and clustering of clinical disorders. For each participant for whom status on all the 15 disorders was defined, the number of identified clinical disorders was counted; this was initially restricted to level 1 disorders, then repeated to include those with level 2 disorders also. The number of disorders per participant was compared between sexes, and by self-rated health using a chi-squared test, grouping those with five or more clinical disorders together. This analysis was then repeated excluding the two disorders (osteoporosis and ECG abnormalities) that depended on the clinic visit for ascertainment. We assessed whether the number of disorders varied according to whether participants had a clinic or home visit, and by current self reported health status. Both weighted and unweighted analyses were again conducted.

To explore further the clustering of level 1 clinical disorders, latent class analysis (LCA) was performed on the level 1 clinical disorder variables. Latent class analysis is a multivariable regression model that describes the relationships between a set of observed dependent variables ('latent class indicators'), in this case the clinical disorders, and an unobserved categorical latent variable, each level of which is referred to as a 'latent class'. For dichotomous latent class indicators, as in the present application, the relationships are described by a set of logistic regression equations [29]. LCA assumes conditional independence of variables within each latent class. The objective is to identify the latent class indicators that best distinguish between classes and to categorise people into their most likely classes given their observed responses [30]. We used a variety of different tools to decide how many classes were required as no single approach is commonly accepted: the Lo-Mendell-Rubin adjusted likelihood ratio test (LRT), the bootstrap LRT, and three information criteria Akaike's Information Criterion (AIC), Schwarz's Bayesian Information Criterion (BIC), and sample size-adjusted BIC (aBIC). The entropy, relative sizes, and meaningful interpretation of the latent classes were also considered. LCA was performed on complete cases only, then repeated using all study members who contributed data on at least one clinical disorder using full information maximum likelihood (FIML) under the assumption of missing at random for comparison [31]. LCA was conducted using Mplus version 6 [32].

The relationship between the latent classes and health status at 36 years was investigated using logistic regression of each latent variable on health status, weighted by the LCA posterior class membership probabilities.

\section{Results}

2657 survey members contributed to the analysis for one or more clinical disorders. Of these, $1104(41.6 \%)$ belonged to the manual social class strata as defined at the initial sampling in 1946. This compares to $2370(44.2 \%)$ of the 5362 study members originally in the cohort.

The commonest disorders were level 1 or level 2 hypertension $(54 \%)$, obesity $(31 \%)$, raised cholesterol $(27 \%)$, and diabetes or impaired fasting glucose $(26 \%)$. Other disorders affecting at least one in ten people were psychiatric disorders $(19 \%)$, chronic respiratory disease $(12 \%)$, cancers $(11 \%)$, osteoporosis $(11 \%)$, cardiovascular disease $(11 \%)$ and renal impairment $(10 \%)$; hypothyroidism $(7 \%)$ and liver disease $(5 \%)$ were less common. There was strong evidence for sex differences in many of the conditions examined. The others showed weak evidence (intermittent claudication, level 2 hyperthyroidism and cancers) or no evidence (anaemia, chronic respiratory disease, level 2 obesity and level 2 renal impairment); the number with level 1 renal impairment was too low to detect a sex difference. Hypertension, raised cholesterol, diabetes, cardiovascular problems, EGG abnormalities and abnormal liver function were more common in men, and psychiatric problems, thyroid disease, osteoporosis, and level 1 i.e. morbid obesity were more common in women (Table 2). The unweighted prevalences were similar for most clinical disorders, but slightly lower for several (results not shown). 
Table 1. Clinical disorders, level 2 and level 1, with data sources and definitions.

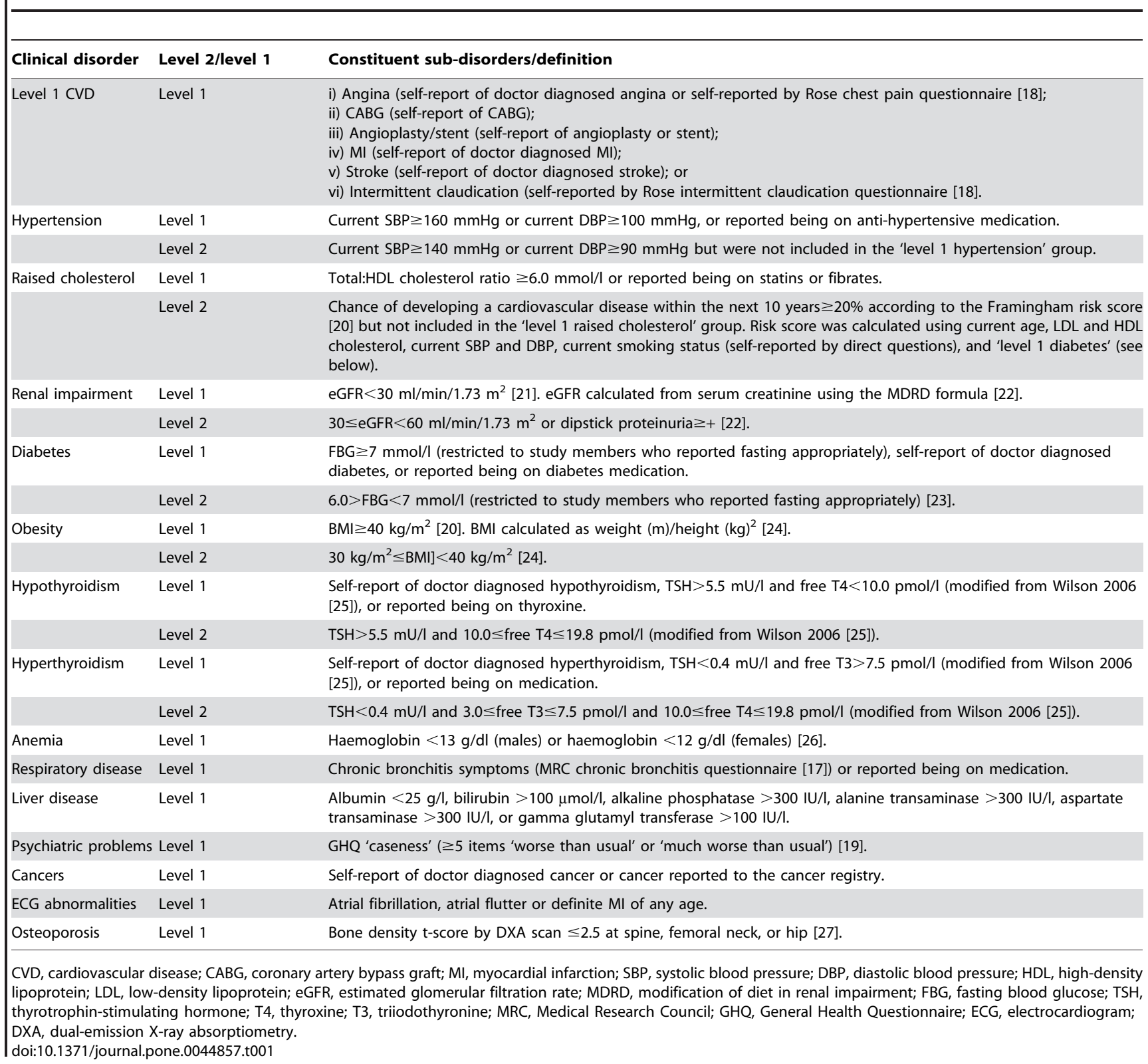

This would be expected if the disorders were more prevalent in the manual social class, of which a smaller fraction were sampled.

\section{Previously undiagnosed disorders}

Table 3 shows the proportions with undiagnosed hypertension, diabetes, thyroid disease, and osteoporosis in the responding sample. Nine percent of the sample had undiagnosed untreated osteoporosis $(79 \%$ of those with osteoporosis), $6 \%$ undiagnosed hypertension (15\% of those with hypertension), 3\% undiagnosed diabetes $(39 \%$ of those with diabetes), and $<1 \%$ undetected thyroid disease ( $8 \%$ of those with thyroid disease). Undiagnosed hypertension and diabetes were commoner in men. Undiagnosed osteoporosis was commoner in women in the full sample; however, within the group with osteoporosis, men were more likely to be undiagnosed $(90 \%)$ than women $(74 \%)$. There were no evidence of sex differences in undiagnosed thyroid disorders. The results from the unweighted analysis were very similar to those in the weighted analysis (results not shown).

\section{Distribution of clinical disorders and relationship to current self reported health}

Table 4 shows the distribution for the number of level 1 clinical disorders for those with information on all 15 or 13 clinical disorders. Thirty percent of the participants were free of all of the 15 level 1 disorders. The results on the larger sample, excluding the conditions only defined at the clinic visit (osteoporosis and ECG abnormalities), were similar, but slightly more participants $(33 \%)$ were disorder free. In both cases the median number of level 1 disorders was 1 (range $0-8$ ), and there was no evidence of sex difference in the number of disorders.

Table 5 shows the number of level 1 and level 2 clinical disorders in those with information on all 15 or 13 clinical disorders. Only $15 \%$ were free of either a level 1 or level 2 
Table 2. Number and percent with level 1 and level 2 clinical disorders in men and women 60-64 years in the MRC National Survey of Health and Development.

\begin{tabular}{|c|c|c|c|c|c|c|c|c|}
\hline \multirow[b]{2}{*}{ Clinical disorder } & \multirow[b]{2}{*}{$\begin{array}{l}\text { Level } 2 / \\
\text { level } 1\end{array}$} & \multicolumn{2}{|l|}{ Males } & \multicolumn{2}{|l|}{ Females } & \multicolumn{2}{|l|}{ Total } & \multirow{2}{*}{$\begin{array}{l}P \text { for diff } \\
\text { between } \\
\text { sexes }\end{array}$} \\
\hline & & $n / N$ & $\%(95 \% \mathrm{Cl})^{\mathrm{A}}$ & $n / N$ & $\%(95 \% \mathrm{Cl})^{\mathrm{A}}$ & $n / N$ & $\%(95 \% \mathrm{Cl})^{A}$ & \\
\hline \multicolumn{9}{|l|}{ Level 1 CVD } \\
\hline Angina & Level 1 & $97 / 1164$ & $9.2(7.3,11.4)$ & $60 / 1263$ & $5.2(3.9,6.9)$ & $157 / 2427$ & $7.1(5.9,8.5)$ & 0.002 \\
\hline CABG & Level 1 & $22 / 1136$ & $2.3(1.4,3.6)$ & $3 / 1209$ & $0.3(0.1,1.2)$ & $25 / 2345$ & $1.3(0.8,2.0)$ & 0.001 \\
\hline Angioplasty/stent & Level 1 & $40 / 1127$ & $3.7(2.6,5.3)$ & $13 / 1205$ & $1.3(0.7,2.4)$ & $53 / 2332$ & $2.5(1.8,3.4)$ & 0.002 \\
\hline $\mathrm{Ml}$ & Level 1 & $59 / 1146$ & $5.8(4.3,7.7)$ & $15 / 1215$ & $1.7(0.9,2.9)$ & $74 / 2361$ & $3.7(2.8,4.7)$ & $<0.001$ \\
\hline Stroke & Level 1 & $34 / 1181$ & $3.1(2.1,4.6)$ & $21 / 1268$ & $2.1(1.3,3.4)$ & $55 / 2449$ & $2.6(1.9,3.5)$ & 0.21 \\
\hline Intermittent claudication & Level 1 & 13/1152 & $1.4(0.8,2.6)$ & $6 / 1235$ & $0.4(0.2,1.1)$ & $19 / 2387$ & $0.9(0.5,1.5)$ & 0.03 \\
\hline Any level 1 CVD & Level 1 & $133 / 1048$ & $13.8(11.5,16.6)$ & $71 / 1093$ & $7.5(5.8,9.7)$ & $204 / 2141$ & $10.6(9.1,12.3)$ & $<0.001$ \\
\hline \multirow[t]{2}{*}{ Hypertension } & Level 1 & 407/1061 & $41.2(37.6,44.8)$ & $348 / 1147$ & $33.0(29.8,36.3)$ & $755 / 2208$ & $36.8(34.4,39.3)$ & 0.001 \\
\hline & Level 2 & $225 / 1061$ & $20.2(17.5,23.2)$ & 188/1147 & $14.9(12.6,17.5)$ & $413 / 2208$ & $17.4(15.6,19.4)$ & 0.005 \\
\hline \multirow[t]{2}{*}{ Raised cholesterol } & Level 1 & 269/960 & $28.0(24.7,31.5)$ & 176/1024 & $18.2(15.5,21.3)$ & $445 / 1984$ & $22.9(20.7,25.2)$ & $<0.001$ \\
\hline & Level 2 & $63 / 785$ & $8.9(6.7,11.6)$ & $5 / 838$ & $0.4(0.1,1.3)$ & $68 / 1623$ & $4.5(3.4,5.9)$ & $<0.001$ \\
\hline \multirow[t]{2}{*}{ Renal impairment } & Level 1 & 2/913 & $0.2(0.0,1.2)$ & $0 / 942$ & $0.0(\mathrm{NA}, \mathrm{NA})$ & $2 / 1855$ & $0.1(0.0,0.6)$ & NA \\
\hline & Level 2 & $95 / 895$ & $11.4(9.1,14.2)$ & 78/932 & $9.4(7.3,12.0)$ & 173/1827 & $10.4(8.8,12.3)$ & 0.25 \\
\hline \multirow[t]{2}{*}{ Diabetes } & Level 1 & $101 / 834$ & $12.8(10.3,15.9)$ & $66 / 907$ & $7.4(5.6,9.7)$ & $167 / 1741$ & $9.9(8.4,11.8)$ & 0.002 \\
\hline & Level 2 & 180/917 & $20.5(17.5,23.9)$ & $113 / 982$ & $11.9(9.7,14.6)$ & 293/1899 & $16.0(14.1,18.1)$ & $<0.001$ \\
\hline \multirow[t]{2}{*}{ Obesity } & Level 1 & 10/1061 & $0.7(0.3,1.5)$ & $31 / 1158$ & $3.0(2.0,4.5)$ & $41 / 2219$ & $1.9(1.3,2.7)$ & $<0.001$ \\
\hline & Level 2 & 290/1061 & $29.3(26.1,32.7)$ & $320 / 1158$ & $29.1(26.0,32.3)$ & $610 / 2219$ & $29.2(26.9,31.5)$ & 0.93 \\
\hline \multirow[t]{2}{*}{ Hypothyroidism } & Level 1 & $20 / 903$ & $1.8(1.0,3.0)$ & 107/969 & $10.9(8.7,13.5)$ & $127 / 1872$ & $6.5(5.3,8.0)$ & $<0.001$ \\
\hline & Level 2 & 18/1000 & $1.5(0.9,2.7)$ & $67 / 1063$ & $6.4(4.8,8.4)$ & $85 / 2063$ & $4.1(3.2,5.2)$ & $<0.001$ \\
\hline \multirow[t]{2}{*}{ Hyperthyroidism } & Level 1 & 6/903 & $0.6(0.2,1.6)$ & $22 / 968$ & $2.7(1.7,4.3)$ & $28 / 1871$ & $1.7(1.1,2.6)$ & 0.003 \\
\hline & Level 2 & $7 / 1000$ & $0.6(0.2,1.5)$ & $17 / 1062$ & $1.5(0.8,2.6)$ & $24 / 2062$ & $1.0(0.6,1.7)$ & 0.09 \\
\hline Anemia & Level 1 & $45 / 999$ & $3.8(2.7,5.5)$ & $48 / 1063$ & $5.1(3.7,7.0)$ & $93 / 2062$ & $4.5(3.6,5.7)$ & 0.23 \\
\hline Respiratory disease & Level 1 & 140/1132 & $12.6(10.4,15.1)$ & $140 / 1223$ & $12.2(10.2,14.6)$ & $280 / 2355$ & $12.4(10.9,14.1)$ & 0.84 \\
\hline Liver disease & Level 1 & $74 / 1002$ & $7.3(5.6,9.5)$ & $37 / 1059$ & $3.3(2.2,4.8)$ & $111 / 2061$ & $5.2(4.2,6.5)$ & 0.001 \\
\hline Psychiatric problems & Level 1 & 137/1047 & $13.5(11.2,16.2)$ & $250 / 1136$ & $23.3(20.4,26.4)$ & $387 / 2183$ & $18.7(16.8,20.7)$ & $<0.001$ \\
\hline Cancers & Level 1 & 108/1169 & $9.6(7.8,11.9)$ & $164 / 1270$ & $12.5(10.5,14.8)$ & $272 / 2439$ & $11.1(9.7,12.7)$ & 0.06 \\
\hline ECG abnormalities & Level 1 & $47 / 785$ & $6.2(4.5,8.6)$ & $14 / 846$ & $2.1(1.1,3.7)$ & $61^{B} / 1631$ & $4.1(3.0,5.4)$ & 0.001 \\
\hline Osteoporosis & Level 1 & $50 / 780$ & $7.3(5.3,10.0)$ & $129 / 853$ & $14.0(11.5,17.0)$ & $179 / 1633$ & $10.9(9.2,12.8)$ & $<0.001$ \\
\hline
\end{tabular}

disorders when 15 clinical disorders were considered, and $16 \%$ were disorder free when 13 clinical disorders were considered. In both cases the median number of level 2 or level 1 disorders was 2 (range 0 to 9 ). Women were somewhat more likely to be disorder free.

The distributions of the number of clinical disorders per study member were broadly similar in the unweighted analysis, though a slightly greater proportion of study members were disorder-free (results not shown). Again, this would be expected if disorders were more prevalent in the manual social class.

Those who had a home visit were less likely to be free from clinical disorders than those who had a clinic visit $(23.3 \% \mathrm{v}$. $34.0 \%, \mathrm{p}=0.02$ for level 1 clinical disorders).

In the total responding sample, $13 \%$ of participants described their health as excellent, $40 \%$ as very good, $32 \%$ as good, $13 \%$ as fair, and $2 \%$ as poor. These ratings were strongly associated with the number of level 1 and level 2 clinical disorders reported $(\mathrm{p}<0.001)$. For example, considering all 15 clinical disorders, $77 \%$ of those with no level 1 or 2 clinical disorders rated their health as excellent or very good and $2 \%$ rated it as fair or poor; but only $31 \%$ of those with 5 or more level 1 or 2 disorders rated their health as excellent or very good and $40 \%$ rated it as fair or poor. Around $50 \%$ of study members with cardiovascular disease or morbid obesity rated their health as fair or poor. These figures were essentially unchanged in the unweighted analysis.

\section{Clustering of clinical disorders and relationship to health status at 36 years}

Level 1 renal impairment was excluded from the LCA as the prevalence was too low. The remaining 14 clinical disorders meant that there were 16384 possible patterns of clinical disorders within 
Table 3. Diagnosed and undiagnosed level 1 clinical disorders.

\begin{tabular}{|c|c|c|c|c|c|c|c|}
\hline \multirow[b]{3}{*}{ Clinical disorder } & \multicolumn{6}{|l|}{$n / N\left(\%^{A}\right)$} & \multirow{3}{*}{$\begin{array}{l}P \text { for diff in } \\
\text { undiagnosed } \\
\text { between sexes }\end{array}$} \\
\hline & \multicolumn{2}{|l|}{ Males } & \multicolumn{2}{|l|}{ Females } & \multicolumn{2}{|l|}{ Total } & \\
\hline & Diagnosed & Undiagnosed & Diagnosed & Undiagnosed & Diagnosed & Undiagnosed & \\
\hline Hypertension & $331 / 1061$ (32.5) & 76/1061 (8.7) & 309/1147 (29.5) & 39/1147 (3.5) & 640/2208 (30.9) & 115/2208 (5.9) & $<0.001$ \\
\hline Diabetes & $58 / 834(8.0)$ & 43/834 (4.8) & 43/907 (5.2) & 23/907 (2.2) & $101 / 1741(6.5)$ & 66/1741 (3.4) & 0.01 \\
\hline Hypothyroidism & 19/903 (1.6) & $1 / 903(0.2)$ & 99/969 (10.2) & 8/969 (0.6) & 118/1872 (6.1) & 9/1872 (0.4) & 0.26 \\
\hline Hyperthyriodism & 5/903 (0.6) & $1 / 903(0.1)$ & 19/968 (2.4) & 3/968 (0.3) & 24/1871 (1.5) & 4/1871 (0.2) & 0.12 \\
\hline Osteoporosis & $5 / 780(0.5)$ & $45 / 780(6.8)$ & 33/853 (3.7) & 96/853 (10.3) & 38/1633 (2.2) & 141/1633 (8.6) & 0.04 \\
\hline
\end{tabular}

${ }^{A}$ Weighted according to original social class-stratified sampling.

doi:10.1371/journal.pone.0044857.t003

the dataset. There was strong evidence that LCA models in which parameters were allowed to differ between males and females provided a better fit to the data than a model which combined both sexes. For both males and females a two class model provided the best fit to the data. Figures 1 and 2 show the latent classes of clinical disorders for males and females respectively using standardised probabilities, which are calculated by dividing the probability of each clinical disorder in each latent class by the observed proportion of study members with that disorder in the sample. For both males and females there was a smaller latent class ( $\mathrm{n}=77$ for men and $\mathrm{n}=112$ for women) in which participants had a high probability of cardio-metabolic disorders (Figure la and 2a). The remaining larger latent class for men $(n=436)$ and women $(n=428)$ had a generally low probability of disorders (Figure $1 \mathrm{~b}$ and $2 \mathrm{~b}$ ). As this larger cluster included participants with no disorders, the analysis was repeated excluding them. This gave essentially the same result, distinguishing those with a high probability of cardio-metabolic disorders from others. Repeating the LCA using all study members who contributed data on at least one clinical disorder using FIML increased the number of study members contributing to the analysis to 1283 men and 1374 women. Two latent classes were again required for both men and women, and the probabilities of each clinical disorder within each latent class were very similar to those in the complete case analysis (results not shown). However, the classes were not as clearly separated (lower entropy) due to the inclusion of study members with reduced information.

In both males and females there was evidence of a trend in the relationship between the latent classes at 60-64 years and health status at age 36 years, with those in the worst health at age 36 more likely to be in the cardio-metabolic latent class almost thirty years later. Of those in the worst of health at 36 years, $25.5 \%$ of men and $30.9 \%$ of women were in the cardio-metabolic latent class at 60-4 years, whereas this was only true for only $13.2 \%$ of men and $14.6 \%$ of women in the best of health almost thirty years earlier $(p=0.02$ and $p=0.003$ respectively). When using the latent classes derived under FIML the magnitude of the associations was very similar (results not shown).

\section{Discussion}

\section{Key findings}

In this survey of common clinical disorders in the first wave of British baby boomers at 60-64 years, we found that most had clinical disorders even though the majority described their general health as good, or better. These disorders were widely spread across the population, with only one in six people being free of all the disorders considered i.e. not requiring any medical interven-

Table 4. Number (percent) of level 1 clinical disorders per study member.

\begin{tabular}{|c|c|c|c|c|c|c|}
\hline \multirow{3}{*}{$\begin{array}{l}\text { Number of clinical } \\
\text { disorders }\end{array}$} & \multicolumn{6}{|l|}{$\mathbf{n}\left(\%^{A}\right)$} \\
\hline & \multicolumn{3}{|c|}{ All clinical disorders $(\max .15)^{B}$} & \multicolumn{3}{|c|}{ Excluding osteoporosis and ECG abnormalities (max. 13) } \\
\hline & Males & Females & Total & Males & Females & Total \\
\hline 0 & $146(26.3)$ & $171(32.4)$ & 317 (29.5) & $194(30.8)$ & $222(34.2)$ & $416(32.6)$ \\
\hline 1 & $178(34.4)$ & $162(29.6)$ & $340(31.8)$ & $206(32.0)$ & 198 (31.6) & $404(31.8)$ \\
\hline 2 & $84(18.2)$ & $114(19.6)$ & $198(18.9)$ & $96(16.1)$ & 115 (17.6) & 211 (16.9) \\
\hline 3 & $63(12.8)$ & $53(10.5)$ & 116 (11.6) & $72(12.5)$ & $58(10.0)$ & $130(11.2)$ \\
\hline 4 & $28(5.2)$ & $24(4.4)$ & $52(4.8)$ & $31(5.5)$ & $22(3.8)$ & $53(4.6)$ \\
\hline $5+$ & $14(3.3)$ & 15 (3.6) & $29(3.5)$ & $16(3.0)$ & $13(2.7)$ & $29(2.8)$ \\
\hline Total & 513 & 539 & 1052 & 615 & 628 & 1243 \\
\hline
\end{tabular}

${ }^{A}$ Weighted according to original social class-stratified sampling.

${ }^{B}$ Range: $0-8$; median: $1 ; P$ value for diff between sexes $=0.46$.

CRange: $0-8$; median: $1 ; P$ value for diff between sexes $=0.60$.

doi:10.1371/journal.pone.0044857.t004 
Table 5. Number (percent) of level 1-level 2 clinical disorders per study member.

\begin{tabular}{|c|c|c|c|c|c|c|}
\hline \multirow{3}{*}{$\begin{array}{l}\text { Number of } \\
\text { clinical } \\
\text { disorders }\end{array}$} & \multicolumn{6}{|l|}{$n\left(\%^{A}\right)$} \\
\hline & \multicolumn{3}{|c|}{ All clinical disorders $(\max .15)^{B}$} & \multicolumn{3}{|c|}{ Excluding osteoporosis and ECG abnormalities (max. 13$)^{c}$} \\
\hline & Males & Females & Total & Males & Females & Total \\
\hline 0 & 69 (11.3) & $92(17.7)$ & $161(14.7)$ & $91(13.8)$ & $117(18.2)$ & $208(16.1)$ \\
\hline 1 & $116(22.5)$ & $128(23.4)$ & $244(23.0)$ & $148(23.9)$ & $160(26.7)$ & $308(25.3)$ \\
\hline 2 & $108(24.1)$ & $128(24.8)$ & $236(24.4)$ & $119(21.8)$ & $150(24.8)$ & $269(23.3)$ \\
\hline 3 & $89(18.0)$ & $83(16.4)$ & $172(17.2)$ & $103(17.1)$ & $90(15.5)$ & $193(16.3)$ \\
\hline 4 & $61(12.6)$ & $50(9.6)$ & $111(11.0)$ & $64(12.1)$ & $52(8.0)$ & $116(10.0)$ \\
\hline $5+$ & $50(11.5)$ & $39(8.1)$ & $89(9.7)$ & $60(11.3)$ & $35(6.8)$ & $95(9.0)$ \\
\hline Total & 493 & 520 & 1013 & 585 & 604 & 1199 \\
\hline
\end{tabular}

${ }^{A}$ Weighted according to original social class-stratified sampling.

${ }^{B}$ Range: 0-9; median: 2; $P$ value for diff between sexes $=0.13$.

CRange: 0-9; median: 2; $P$ value for diff between sexes $=0.03$.

doi:10.1371/journal.pone.0044857.t005

tion; this distribution was strongly related to self reported health. We identified a latent class of cardio-metabolic disorders, covering one in five of the population; this class was more likely to have had poor health as young adults.

\section{Strengths and limitations of the study}

As with all longitudinal studies NSHD has suffered from attrition. However, this is a large, national cohort that remains reasonably representative of the British born population. The NSHD study population is however all white, and therefore our findings cannot be extrapolated to the non-White British population. Non-White people make up $9 \%$ of the current population of England and Wales but they generally have a younger age profile, and only $5 \%$ of the population $60-64$ years are non-White [33].

Some of our disorders are defined only on self-report of a doctor's diagnosis of disease. However, self-reports are generally

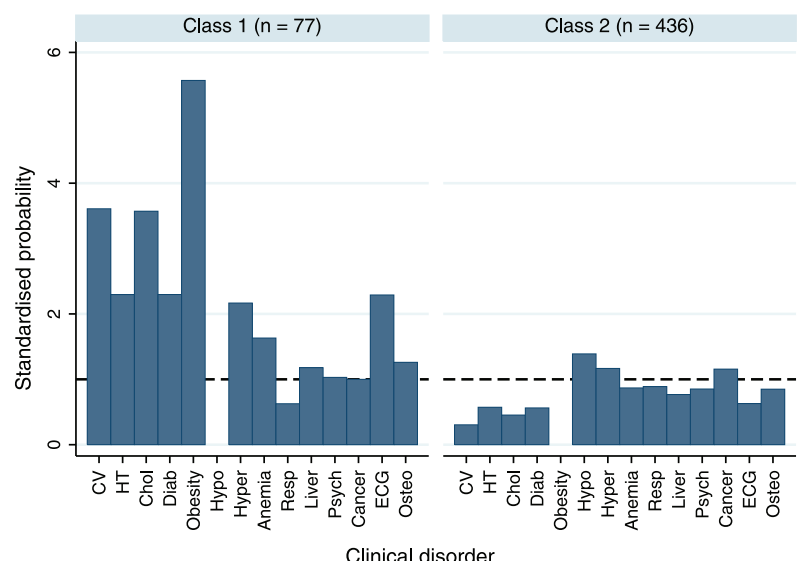

Figure 1. 1a and 1b. Latent classes of level 1 clinical disorders (excluding renalimpairment) in males $(n=513)$. Standardised probabilities are calculated by dividing the probability of each clinical disorder within each latent class by the observed proportion of study members with that disorder in the sample.

doi:10.1371/journal.pone.0044857.g001 found to be reasonably accurate when compared with medical records in cases of definite diagnoses [34,35]. In the NSHD we sent questionnaires to GPs asking them to confirm self reports of chronic disease; for diabetes we found over 90\% agreement [36]. We only measured blood pressure on one occasion so we may have overestimated the amount of previously undiagnosed hypertension, since in practice a diagnosis of hypertension is based on at least two readings taken at different times.

Rare disorders or common disorders in which confirmatory clinical assessments were not possible, and treatment is nonspecific e.g. arthritis, were not included. Hence this study estimated the extent of common clinical disorders rather than the full extent of health problems in this cohort at retirement age.

Although our analysis of the clustering of clinical disorders within study members was presented for complete cases only, obtaining very similar results using all study members who contributed data on at least one clinical disorder suggested that

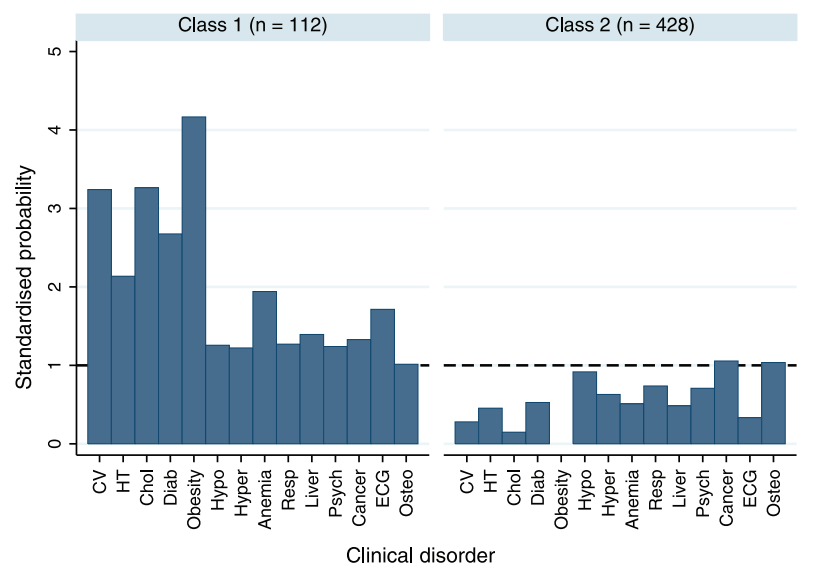

Figure 2. 2a and 2b. Latent classes of level 1 clinical disorders (excluding renal impairment) in females $(n=540)$. Standardised probabilities are calculated by dividing the probability of each clinical disorder within each latent class by the observed proportion of study members with that disorder in the sample.

doi:10.1371/journal.pone.0044857.g002 
significant bias was not introduced.

\section{Comparison of our findings with other published studies}

The proportions observed for cancers, diabetes, thyroid disease, obesity and psychiatric problems were similar to those in other studies, as were those for previously undiagnosed thyroid disorders and diabetes [20,22,36-44]. The major difference between our results and other reported studies was in relation to hypertension, the NSHD population had noticeably more people with hypertension than reported in HSE 2009 [37]. Our findings on renal impairment are not directly comparable with the other UK data $[37,42]$ as these studies report CKD stages 3-5 (NICE 2008) [21] which exclude people with proteinuria but eGFR $>60 \mathrm{ml} / \mathrm{min} /$ $1.73 \mathrm{~m}^{2}$ from being classified as having renal impairment. In NSHD the proportion with of CKD stages 3-5 is slightly lower than for the other studies [37,42]. However people with proteinuria alone do have a higher CVD and renal progression risk [22], and the NSHD identifies this group for the first time in a British general population. We found somewhat fewer MIs, strokes, and better respiratory function than has been reported in cross-sectional studies in people aged 64-69 in England [38], but this is probably accounted for by the age difference. NSHD women had somewhat less osteoporosis than estimated by WHO [27]; this may be an underestimation because participants who attended the clinics (where bone densitometry was available) had fewer clinical disorders than the people seen at home visits.

Undetected disorders. There was a high proportion of undetected osteoporosis in men and women. This is a condition that is not screened for as population screening is not cost effective [44]. National guidelines [44] do encourage case finding but this may not be widely applied and awareness of the problem in men is not high [45]. This may improve in the future with osteoporosis being introduced into the Quality Outcomes Framework in 2012. Undetected diabetes within the responding population was more frequent than found in the English Longitudinal Study of Ageing [39]; almost two fifths of those with diabetes had not been previously diagnosed. There was very little undetected thyroid disease, as found in the Birmingham Elderly Thyroid Study (BETS) [25]. This may be explained by GP awareness of thyroid disorders conditions and their success in case finding. The levels of undiagnosed hypertension and diabetes should reduce once the proposal for Vascular Screening system in the over 45 year-olds comes on-line in general practice [7].

Multiple disorders and clustering of disorders. In the Newcastle cohort study of those aged 85 years and older, nobody was disorder-free. This study also included heart failure, dementia and Parkinson's disease but we did not, as these conditions are rare in 65 years olds. Unlike our study, the authors found that women had more clinical disorders than men [9].

The picture we outline here is in many ways a best case scenario. It may be that that people born in the immediate postwar period are healthier than earlier or later born people because they have spent their whole lives within the post-war welfare state and only later experienced exposure to obesogenic lifestyles. There are a number of other conditions which we have not included, e.g. sarcopenia and osteopenia, that are as yet not monitored or treated but, given shifting boundaries, could easily become defined as clinical disorders [4]. We have not followed recent proposals for extending risk factors further back into the normal distribution identifying 'prehypertension' ( $\mathrm{SBP} \geq 120 \mathrm{mmHg}$ ); impaired fasting glycaemia as beginning at $5.6 \mathrm{mmol} / \mathrm{l}$; and borderline risk of LDL as beginning at $3.6 \mathrm{mmol} / \mathrm{l}$. In the US population Kaplan and Ong [46] estimate that such categorization of these three risk factors alone would result in $97 \%$ of the population being under medical surveillance.

The study of patterns of multi-morbidity is relatively new field. There are few studies that have examined the way conditions cluster within groups of patients. One in the USA examined the records of 1.3 million primary care patients cared for by the Veterans Health Care System with two or more co morbidities and categorized 45 health conditions [47]. They reported that $83 \%$ of their sample fell into their metabolic cluster. An Australian study in working age adults [48] found that health conditions do not cluster neatly into organ or body systems as has been assumed by methods underpinning the Cumulative Index Rating Scale [49] and they identified 6 independent clusters of disorders.

\section{Implications}

Few people are without a clinical disorder on reaching retirement age. This highlights two sets of problems - the first is conceptual, related to our changing definition of disease and to current theories of ageing; and the second is pragmatic, and concerns the workload of health services going forward.

We have increasingly moved from a diseased-based model of medicine where doctors reacted to signs and symptoms presented by the patient, to a more proactive risk based model in which many clinical disorders are the end of a distribution of biological attributes, and are detected on the basis of case finding. This move has been driven by the success of epidemiological studies in identifying risk factors, and demonstrating effective interventions to reduce risk and to treat diseases early. Certainly this more proactive approach has coincided with a marked reduction in mortality rates for cardiovascular disease [50], although some would argue that the relationship between the two is not causal [51].

Individuals have different patterns of ageing. Such patterns have been postulated as; 'survivors' who live with extended morbidity due to age related disease diagnosed before old age; 'extenders' who live longer than expected without problems and have a shorter period of disability before death; and 'escapers' who attain very old age without disease $[49,50]$. As life expectancy increases there is a debate about whether we are facing a compression of morbidity, with people both living longer and having a longer period of healthy life (more extenders and escapers) or an expansion of morbidity with people living longer but having little or no increase in healthy life (more survivors). Alternatively, we may be facing some intermediate state involving more people living longer but with less severe morbidity (more survivors but with less disability). It has been proposed that ageing research should focus on extenders and escapers, to identify factors related to compression of morbidity and to avoid a pandemic of disability [52,53], and not just on age-related disease.

However, our research, and that emerging from the Netherlands [54], suggest that only a small minority of people in Western populations are 'escapers' or 'extenders', even by retirement age. Data from the Longitudinal Aging Study Amsterdam do not support the compression of morbidity scenario [54], and a study across OECD countries shows inconsistent results [55]. Thus ageing with clinical disorders may become the norm, and it might better to speak in terms of health relative to others of the same age. This situation is prompting new formulations of the meaning of health, especially in relation to older people, which focus on the individual's ability to adapt and self manage physically, psychologically and socially to their changing internal and external environment [56,57].

With our changing definitions of disease and the ageing population comes a significant load on the health services, 
especially general practice, in terms of monitoring and treatment. Not only is general practice delivering its traditional reactive role but it is increasingly expected to deliver the preventive care part of the public health agenda. The effect on workload is already being seen. Over the past decade patient consultation rates per patient in England and Wales have increased over 40\% (from 3.9 in 1995/6 to 5.5 per patient per year 2008/9) [58], and this is also being seen in the other OECD countries [59].

The commonest conditions in this study are cardio-metabolic, cancers and osteoporosis which share common upstream causes in the nutritional deterioration of the nation's diet and sedentary behaviour. Unless we are able to tackle successfully these upstream causes on a societal level, we will be left with treating the effects in individuals, either monitoring and trying to change behaviour or prescribing medications. Our demonstration that health status at retirement is strongly associated with health status almost thirty years earlier suggests that a high risk group could be identified that may benefit from individual level interventions that start earlier than middle age. The current structure of the clinical care is still based on the disease model, and may be overwhelmed by the demands of proactive care. The purpose of this paper is not to give solutions to these problems, but to highlight the current position, and emphasize that the proportion of the population 'under the doctor' will only increase given an ageing population and diagnostic creep.

\section{References}

1. Illich I (1975) Medical nemesis: the expropriation of health. London: Calder and Boyars.

2. Ebrahim S (2001) The medicalization of old age should be encouraged. BMJ 324:861-863.

3. Tinetti M and Fried T (2004) The end of the disease era. Am J. Med 116:179185.

4. Moynihan R (2011) A new deal on disease definition. BMJ 342:1054-56.

5. Martin C (2010) Why medicine is overweight. BMJ 2010 340:c2800.

6. Ferrucci L, Studenski S (2012) Clinical problems of aging. In: Longo DL, Fauci AS, Kasper DL, Hauser SL, Jameson JL, Loscalzo J, editors. Harrison's Principles of Internal Medicine. $18^{\text {th }}$ ed. New York, NY: McGraw-Hill. pp. 570585.

7. Department of Health (2008) Putting prevention first-vascular checks: risk assessment and management. London: Department of Health Publications.

8. Wald NJ, Law MR (2003) A strategy to reduce cardiovascular disease by more than $80 \%$. BMJ 326:1419-23.

9. Collerton J, Davies K, Jagger C, Kingston A, Bond J, et al. (2009) Health and disease in 85 year olds: baseline findings from the Newcastle $85+$ cohort study. BMJ 339:b4904.

10. NHS Information centre (2012) The Health Survey for England. Available: http://www.ic.nhs.uk/hse. Accessed 20 April 2012.

11. The Scottish Government (2012) The Scottish Health Survey. Available http:// www.scotland.gov.uk/Topics/Statistics/Browse/Health/scottish-health-survey. Accessed 20 April 2012.

12. Centres for Disease Control and prevention (2012) National Health and Nutrition Survey. Available: http://www.cdc.gov/nchs/nhanes.htm. Accessed 20 April 2012.

13. Wadsworth MEJ, Kuh D, Richards M, Hardy R (2006) Cohort profile: the 1946 birth cohort (MRC National Survey of Health and Development). Int J Epidemiol 35:49-54.

14. Kuh D, Pierce M, Adams J, Deanfield J, Ekelund U, et al. (2011) A Cohort profile: Updating the cohort profile for the MRC National Survey of Health and development: a new clinic based data collection for ageing research. Int J Epidemiol 40(1):e1-9.

15. Office for National Statistics (2009) National population projections-2008 based. Available:http: / / www.ons.gov.uk/ons / search/index. html ?pageSize $=50 \&$ newquery $=$ age + sex + projections +2009 . Accessed $10 \mathrm{Janu}-$ ary 2012 .

16. Joint Committee of the Royal College of Obstetricians and Gynaecologists and the Population Investigation Committee (1948). Maternity in Great Britain. Oxford University Press, London.

17. Medical Research Council on the Aetiology of Chronic Bronchitis (1960) Standardised questionnaire on respiratory symptoms. BMJ 2:1665.

\section{Acknowledgments}

The authors are grateful to NSHD study members who took part in this latest data collection for their continuing support. We thank members of the NSHD scientific and data collection teams at the following centres: MRC Unit for Lifelong Health and Ageing, MRC Human Nutrition Research, Cambridge; MRC Epidemiology Unit, Cambridge; MRC Epidemiology Resource Centre, Southampton; Welcome Trust (WT) Clinical Research Facility (CRF) Manchester, the Manchester Heart Centre, and the Department of Clinical Radiology at the Central Manchester University Hospitals NHS Foundation Trust; WTCRF, Medical Physics and the Department of Cardiology at the Western General Hospital in Edinburgh; WTCRF, Department of Nuclear Medicine and the Department of Cardiology at University Hospital Birmingham; WTCRF and the Department of Nuclear Medicine at University College London Hospital; CRF, the Department of Medical Physics and the Department of Cardiology at the University Hospital of Wales; CRF and Twin Research Unit at St Thomas' Hospital London; Vascular Physiology Unit, Institute of Child Health, London; National Heart and Lung Institute, Imperial College London; Divisional of Cardiovascular \& Medical Sciences, Western Infirmary, Glasgow; Cardiovascular Institute, Sahlgrenska Academy, Gothenburg University.

We also thank Professor Jayne Franklyn from the University of Birmingham, Dr Joan Trowel (retired hepatologist) and Dr Ian Halsall who provided expert advice on blood analyte levels.

\section{Author Contributions}

Conceived and designed the experiments: DK MP JA PM DN MR RH. Performed the experiments: AW DK MR MP NSHD Data Collection Team. Analyzed the data: PM JA WN AS RS. Wrote the paper: MP DK. Revised the manuscript: MP RS DN JA AS WN PM AW MR RH DK.

18. Rose G, McCartney P, Reid DD (1977) Self-administration of a questionnaire on chest pain and intermittent claudication. Br J Prev Soc Med 31: 42-8.

19. Goldberg DP, Hillier VF (1979) A scaled version of the General Health Questionnaire. Psychol Med 9:139-145.

20. Wilson PW, D'Agostino RB, Levy D, Belanger AM, Silbershatz H, et al. (1998) Prediction of coronary heart disease using risk factor categories. Circulation 97: 1837-47.

21. NHS. National Institute for Clinical Excellence (2008) Early identification and management of renal disease in adults in primary and secondary care. Clinical guidelines, CG73. Available: http://publications.nice.org.uk/chronic-kidneydisease-cg73. Accessed 17 April 2012.

22. Levey AS, de Jong PE, Coresh J, El Nahas M, Astor BC, et al. (2011) The definition, classification and prognosis of chronic kidney disease: a KDIGO Controversies Conference report. Kidney Int 80: 17-28.

23. World Health Organization (2006) Definition and diagnosis of diabetes mellitus and intermediate hyperglycaemia. Geneva: World Health Organization.

24. World Health Organization (2004) Obesity: preventing and managing the global epidemic. Geneva: World Health Organization.

25. Wilson S, Parle JV, Roberts LM, Roalfe AK, Hobbs FD, et al. (2006) Prevalence of subclinical thyroid dysfunction and its relation to socioeconomic deprivation in the elderly: a community-based cross-sectional survey. J Clin Endocrinol Metab 91: 4809-16.

26. World Health Organization (2008) Worldwide prevalence of anaemia 19932005: WHO global database on anaemia. Geneva: World Health Organization.

27. World Health Organization (1994) Assessment of fracture risk and its application to screening for postmenopausal osteoporosis. Geneva: World Health Organization.

28. Kuh D and Wadsworth MEJ (1993) Physical health at 36 years in a British national birth cohort. Soc Sci Med 37: 905-916.

29. Muthén LK, Muthén BO (1998-2007) Mplus User's Guide. Sixth Edition. Los Angeles, CA: Muthén \& Muthén.

30. Nylund KL, Asparouhov T, Muthen BO (2007) Deciding on the Number of Classes in Latent Class Analysis and Growth Mixture Modeling: A Monte Carlo Simulation Study. Struct Equ Modeling 14(4): 535-69.

31. Little RJA, Rubin DB (2002). Statistical Analysis With Missing Data. Wiley: New York.

32. Muthén \& Muthén (2010). Mplus statistical software, release 6. Muthén \& Muthén: Los Angeles, CA.

33. Insighteast (2001) Census table S101 Sex and Age by Ethnic Group (2001 Census). Available:http://insighteast.org.uk/viewResource.aspx?id = 11003. Accessed 10 January 2012.

34. Tisnado DM, Adams JL, Liu H, Cen WP, Hu FA, et al. (2006) What is the concordance between the medical record and patient self-report as data sources for ambulatory care? Med Care 44:132-40. 
35. Klungel OH, de Boer A, Paes AH, Seidell JC, Bakker A (1999) Cardiovascular diseases and risk factors in a population-based study in The Netherlands: agreement between questionnaire information and medical records. Neth J Med 55:177-83.

36. Pastorino S and Pierce M (2011) Validation of self-reported questionnaires for diagnosis of diabetes in a British birth cohort. Diabet Med 28:32-203.

37. NHS Information Centre (2009) Health Survey for England- 2009: Health and lifestyles. Available:http://www.ic.nhs.uk/pubs/hse09report. Accessed 10th January 2012.

38. NHS Information Centre (2005) Health Survey for England 2005. The health of older people. Available: http://www.ic.nhs.uk/webfiles/publications/hseolder/ vol2.pdf .Accessed 10th January 2012.

39. Pierce MB, Zaninotto P, Steel N, Mindell J (2009) Undiagnosed diabetes-data from the English Longitudinal Study of Ageing. Diabet Med Jul:26(7):679-85.

40. Vanderpump MP, Tunbridge WM, French JM, Appleton D, Bates D, et al. (1995) The incidence of thyroid disorders in the community: a twenty-year follow-up of the Whickham Survey. Clin Endocrinol 43:55-68.

41. McManus S, Melter H, Bughra T, Bewbbington P, Jenkins R (2007). Adult psychiatric morbidity in England 2007. Results of a household survey. Available:http://www.ic.nhs.uk/pubs/psychiatricmorbidity07. Accessed 10th January 2012.

42. Stevens PE, O'Donoghue DJ, de Lusignan S, Van Vlymen J, Klebe B, et al. (2007) Chronic kidney disease management in the United Kingdom: NEOERICA project results. Kidney Int 72(1):92-9.

43. NHS Information Centre (2007) The Health Survey for England 2007, Vol 1. Healthy lifestyles, Knowledge and Behaviour. Available:http://www.ic.nhs.uk/ webfiles/publications/HSE07/HSE\%2007-Volume\%201.pdf. Accessed 10 January 2012.

44. Royal College of Physicians (1999) Osteoporosis: guidelines for prevention and treatment. Royal College of Physicians, London, UK, pp 63-70.

45. Gielen E, Vanderschueren D, Callewaert F, Boonen S (2011) Osteoporosis in men. Best Pract Res Clin Endocrinol Metab 25:321-35.

46. Kaplan R M and Ong M (2007) Rationale and Public Health Implications of Changing CHD Risk Factor Definitions. Annual Review of Public Health 28; 321-44

47. Cornell JE, Pugh JA, Williams JW, Kazis L, Lee AFS, et al. (2007) Multimorbidity clusters: Clustering binary data from multimorbidity clusters:
Clustering binary data from a large administrative medical database. Applied Multivariate Research 2007, 12:163-182.

48. Holden l, Scuffham PA, Hilton MF, Muspratt A, Ng S, et al, (2011) Patterns of multi-morbidity in working Australians. Population Health Metrics 9:15.

49. Huddon C, Fotin M, Vanasse A (2005) Cumulative Illness Rating Scale was a valid and reliable index in a family practice context. J Clin Epidemiol 58:603608.

50. O'Flaherty M, Ford E, Allender S, Scarborough P, Capewell S (2008) Coronary heart disease trends in England and Wales from 1984 to 2004: concealed levelling of mortality rates among young adults. Heart 94:178-81.

51. Kaplan GA, Baltrus PT, Raghunathan TE (2007) The shape of health to come: prospective study of the determinants of 30-year health trajectories in the Alameda County Study. Int J Epidemiol 36:542-48.

52. Fries JF, Bruce B, Chakravarty E (2011) Compression of morbidity 1980-2011: a focused review of paradigms and progress. J Aging Res 2011:261702.

53. Kivimaki M and Ferrie J (2011) Epidemiology of healthy ageing and the idea of more refined outcome measures. Int J Epidemiol 40:845-847.

54. Deeg D. New myths about ageing. About the growth of medical knowledge and its societal consequences. Chapter $\mathrm{x}$ in McDaniel SA, Zimmer Z, editors. Global Ageing in the Twenty-First Century: Challenges, Opportunities and Implications. Farnham, Surrey, UK: Ashgate Publishing. In press.

55. Lafortune G, Balestat G and the Disability Study Expert Group Members (2007) Trends in severe disability among elderly people: assessing the evidence in 12 OECD countries and the future implications. OECD Health working papers no. 26. Paris:OECD.

56. von faber M, Bootsma-vander Wiel A, van Excel E, Gussekloo J, Lagaay Am, et al. (2001) Successful ageing in the oldest old. Who can be characterized as successfully aged? Arc Int Medicine 161:2694-700.

57. Huber M, Knottnerus JA, Green L, van der Horst H, Jadad AR, Kromhout D, et al. (2011) How should we define health? BMJ 343:4163.

58. Hippsley-Cox J and Vinogardova Y (2009) Trends in consultation rates in general practice 1995/6 to 2005. Available:http://www.ic.nhs.uk/webfiles/ publications/gp/Trends_in_Consultation_Rates_in_General_Practice_1995_ 96_to_2008_09.pdf. Accessed 11 January 2012.

59. OECD (2011) Consultations with doctors. In: OCED, health at a glance 2011:OECD indicators, OECD publishing. doi: 10.1787/health_glance-201129-en 